\title{
Physiological Basis of Cerebellar Dysmetria
}

\author{
JOHN T. MURPHY, HON. C. KWAN, WILLIAM A. MacKAY, AND YIU C. WONG
}

SUMMARY: A primary control system for the arm position is formulated. The hypothesis that the cerebellum is a part of the system controller is checked by studying the nerve cells responses in the cerebellum, and motor cortex, to natural activation of muscular receptors. The results show that the cerebellum receives feedback information related to the speed of these receptors. The discussion concentrates on how the interruption of this feedback may result in excessive oscillations to instability. These observations are the base for evaluating how the cerebral lesions produce dismeasurements.

RÉSUMÉ: Un système élémentaire de contrôle pour la position du bras est formulé. L'hypothèse que le cervelet fait partie du contrôle du système est vérifiée par l'étude des réponses des cellules nerveuses dans le cervelet et le cortex moteur à l'activation naturelle de récepteurs musculaires. Les résultats montrent que le cervelet reçoit l'information "feedback" reliée à la vitesse de ces récepteurs. La discussion se concentre sur comment l'interruption de ce feedback peut résulter en des oscillations excessives à l'instabilité. Ces observations servent de base pour évaluer comment les lésions cérébrales produisent des dysmétries.

From the Department of Physiology, University of Toronto, Ontario, Canada.

Reprint requests to: Prof. J. T. Murphy, Dept. of Physiology, University of Toronto, Medical Sciences Building, Toronto, Ontario, M5S 1A8, Canada.

\section{INTRODUCTION}

The careful, highly detailed descriptions of the consequences of cerebellar damage due to gunshot injuries by Gordon Holmes (1917) have had considerable influence on the teachings of modern neurology. Most current textbooks adopt the classification of cerebellar signs cited by Holmes which include ataxia, the rebound phenomenon, and adiadochokinesis as important manifestations of cerebellar disease. Holmes further subclassified ataxia into several parts, including decomposition of movement, asynergia, tremor during movement, and dysmetria. Each of these signs can be elicited by a specific test or tests, and is thus empirically useful for diagnostic purposes. However, the use of such descriptive terms has not hastened the development of a conceptual framework concerning cerebellar function. Quite clearly, for purposes of thinking about cerebellar dysfunction, as opposed to describing it, some simplifications are required. Holmes appreciated this fact very well and made an attempt in his later summary paper (1939) to make a more fundamental classification of the disturbances in function caused by cerebellar disease. This attempt was limited, in large part because Holmes did not have access to a satisfactory theoretical foundation in which to place the data from his clinical observations.

The emergence of two theoretical developments in the 1940s allowed some initial simplifications to be made. These were firstly control systems theory, which received impetus initially from military requirements, and secondly cybernetics (Wiener, 1948), which encouraged the application of systems theory to a general class of prob- lems in many fields including biology. Control theory allows one to consider the behaviour of a system in terms of inputs and outputs. Generally one can identify and measure both input and output parameters. In the case of neurological disorders of movement control of a limb, position and more precisely angular position of the limb part can be considered as the controlled output variable. The axis of rotation in this case is the skeletal joint about which the angular movement of the limb part occurs. The input or reference signal may be derived directly from a present external signal, or indirectly from a previous external signal by a spatio-temporal mapping of that signal in neuronal activity (engram).

In his consideration of disorders of voluntary movement in the Croonian Lectures (1922), Holmes noted that "disturbance in the range of movement is one of the most frequent disorders in cerebellar disease". He cited dysmetria, errors in direction, errors in rate and kinetic tremor as prominent manifestations of the disturbance. For purposes of simplification we will use the most general of these terms, dysmetria, to describe the clinical observation. The term means abnormal measurement. In the present context we may consider that position is the parameter being measured and controlled. Muscular forces are adjusted to effect the control of position. Each of the other clinical phenomena cited by Holmes (kinetic tremor, errors in rate and direction) can be considered consequences of dysmetria.

\section{Elementary Control Theory}

In many experimental situations in physiology, a subsystem is functionally disconnected from the rest 


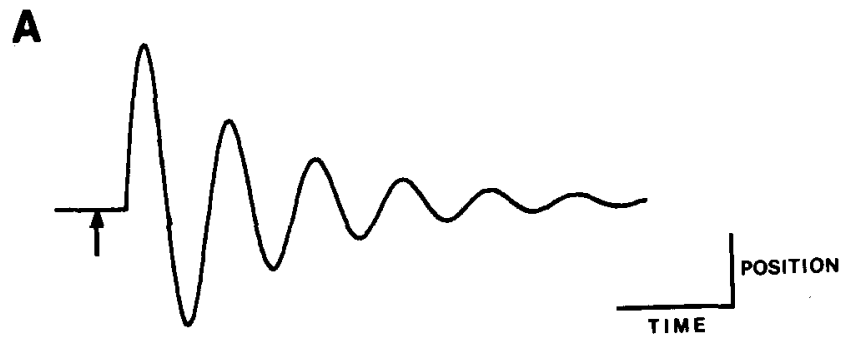

B

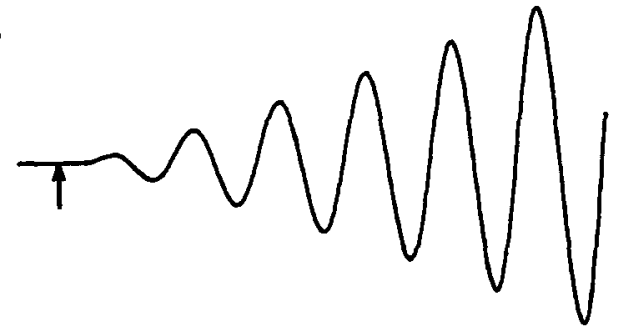

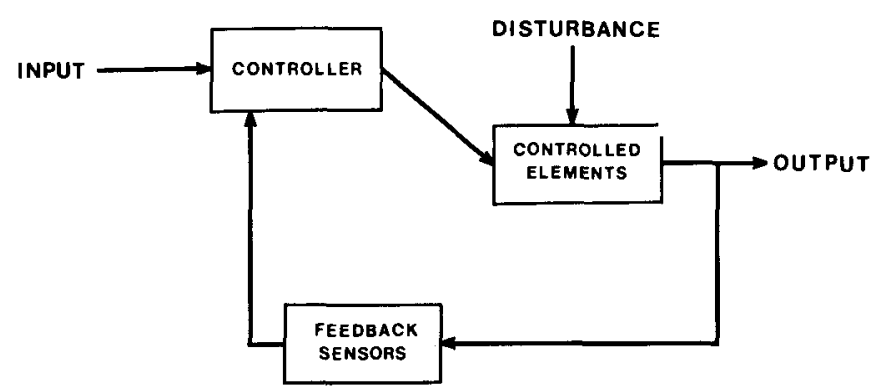

Figure 2-A generalized feedback control system.

Figure 1-A and B. Outputs of a stable (A) and unstable (B) position control system. Arrows indicate time of onset of reference input or disturbance. of the system. This procedure, which is essential to the detailed study of the subsystem, places the whole system in an "open-loop" situation. The intact, normally functioning organism, is considered to be in a "closed-loop" state. Thus the presence of feedback is that characteristic of closed-loop systems which distinguishes them from open-loop systems. In formal control theory, feedback is specifically that property of a closed-loop system which allows the output (which may be any controlled variable of the system) to be compared with the input to the system. This enables a control action to be formulated as some appropriate function of both the input and the output.

In general, simple closed-loop feedback systems tend to be unstable, i.e. they oscillate when perturbed. Intuitively one may consider this as being due to time delays in the system, which results in the input being modified not by the current output, but by an earlier output. The stability or instability of a system is determined by its response to inputs or disturbances. This concept is illustrated in figure 1. The output of a stable system shows oscillations in response to an input, which are damped over time (Fig. 1A). In an unstable system, the oscillations increase with time (Fig. 1B). The degree of relative stability or instability can, if desired, be measured quantitatively.

It is evident that instability is an undesirable characteristic of a system. Yet as indicated above a simple feedback system such as that diagrammed in figure 2 , in which a single variable (i.e. position) is controlled, can be unstable and may produce oscillations of the output in response to either changes in the input or externally applied disturbances. In engineering design problems, three procedures have been effective in stabilizing or at least reducing the degree of instability in control systems. The first is to make adjustments in the single feedback loop which change the loop-time and/or the gain (output/input) of the system. The second is to introduce additional feedback loops with different loop-times and/or processing characteristics than the first. This will result in two changes: firstly a distribution of feedback loop-times, and secondly a change in gain of the system. Either or both of these changes may reduce instability. Finally one may introduce information about the first time derivative of the output variable being controlled, which in the case of position is velocity. Velocity feedback will introduce a phase lead which can compensate for the time delays in the feedback (Stein, 1974). It appears from our recent experimental studies that the brain is arranged in such a way as to utilize each of the latter two principles for movement control and that the cerebellum plays an important role in this regard.
Limiting and Defining the Problem

Restrictions will be placed on the ensuing discussion in order to limit the topic. Some degree of somatotopy is present in the cerebellum as demonstrated both anatomically (Brodal, 1967; Voogd et al., 1969; Grant, 1962) and physiologically (Murphy et al., 1973b; Oscarsson, 1967). The studies to be described are limited to projections from forelimb which are carried to lobule $\mathrm{V}$ of the pars intermedia of the anterior lobe, and also to lobule VIII of the paramedian lobe. In addition, convergence from multiple receptor modalities located within a functional unit of a limb to the same general regions of the cerebellum is also well established (Eccles et al., 1971; Murphy et al., 1973b); however the evidence presented in the following section will deal only with information arising from intramuscular receptors. Before posing questions about feedback we must identify the principal elements of the biological control system under consideration. It must be recognized that there is no absolute or "correct" formulation of a system; the only constraint is that the elements of the system must be defined. This is done in figure 3.

The controlled variable is (angular) position of the limb part. The controller for this system is included within the dashed lines (Fig. 3). We may draw on accumulated knowledge concerning structure and function to show well-established 


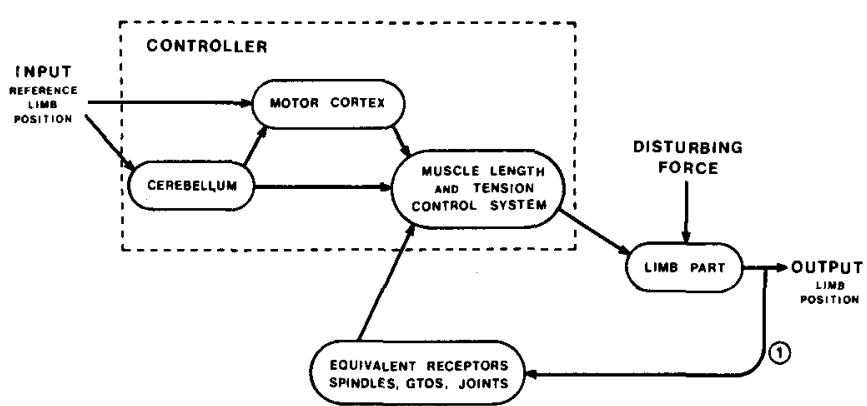

Figure 3-A limb position control system. Equivalent receptors are the sensors for feedback loop (1).

\section{MUSCLE DISPLACEMENT}

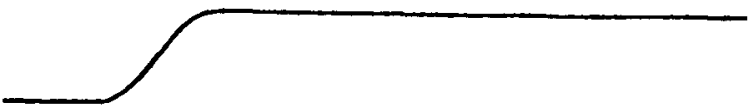

INTERPOSITUS

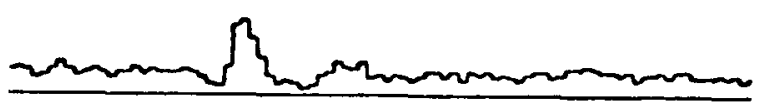

MOTOR CORTEX
Figure 4-Responses of neurons to $1 \mathrm{~mm}$ stretch of single forelimb muscle. Total time for PSTHs, $128 \mathrm{msec}$. Time marker below motor cortex PSTH: $10 \mathrm{msec}$. Bin sizes: 1 msec.

linkages in this block diagram. We define the motor cortex and cerebellum as being the principal brain elements in the controller for this system. Alpha and gamma motoneurons determine muscle activity and ultimately the output of the system: position of a limb part. These motoneurons (in brainstem or spinal cord) are included within the muscle length and tension control system. As motoneuron activity will not be considered in the following discussion, the latter control system has been "collapsed" in figure 3. Suitable expansions may be found in the work of Houk and Henneman (1974). Many other brain elements participate; however in most instances their role has remained unclear and for present purposes they may be considered part of the reference input. The existence of a feedback loop from muscle and joint receptors to spinal motoneurons has been demonstrated exhaustively in physiological studies in the past two and one-half decades (Eccles, 1964; Matthews, 1972). This is designated as a feedback loop 1 in figure 3 .

\section{Do Multiple Feedback Loops Exist?}

Having defined the control system and established from previous knowledge the presence of a primary feedback loop, we directed our experiments to the above simple question. The experiments involved natural stimulation of intramuscular stretch receptors by controlled stretching of the severed distal tendon of single muscles controlling wrist and digit position in the cat forelimb. Local and regional anaesthesia were substituted for general anaesthesia to avoid the distorting effects of the latter on central neuronal networks. Control experiments were done to ensure that only muscle spindles and Golgi tendon organs (GTOs) were activated by the stimulations. These recordings were made from single neurons in the cerebellum and motor cortex. Cells were identified both by physiological criteria and by histological reconstruction of their location. Statistical analysis of neuronal activity was carried out by computer to test validity of conclusions. Feedback loops were opened experimentally by blocking transmission at neuromuscular junctions with gallamine triethiodide.

Under these conditions the recordings in the cerebellum and in the motor cortex yielded unequivocal evidence that multiple feedback loops exist in this control system. Clusters of neurons responsive to natural activation of stretch receptors in muscle were found in somatotopic groupings in the pars intermedia of the cerebellar cortex (Murphy et al., 1973), in the intermediate (interpositus) cerebellar nucleus (Mackay and Murphy, 1974; Kawaguchi and Ono, 1974) and in the forelimb part of the motor cortex (Murphy et al., 1975; Lucier et al., 1975). An example of a neuron in the interpositus nucleus and one in the motor cortex responding to muscle stretch is shown in Figure 4. The presence of feedback to supraspinal structures, in addition to the primary feedback loop to motoneurons shown in figure 3 , by itself ensures a spread of time delays in the feedback, which may reduce instability as indicated earlier. In addition, studies involving latency measurements and cooling of the cerebellar cortex or nuclei show that there are two independent feedback loops to the motor cortex, each with distinct loop times (Murphy et al., 1975). A fast loop probably uses pathways through the dorsal columns and their nuclei, the medial lemniscus and ventroposterolateral thalamus (Rosen, 1972). The feedback to the cerebellum is part of a slower pathway to the motor cortex, involving interpositus nucleus of the cerebellum and ventrolateral thalamus. Moreover interpositus can influence motoneurons through subcortical projections via the red nucleus and 
A

INPUT

EOC DISPLACEMENT

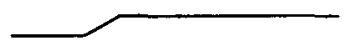

MOTOR CORTEX NEURON

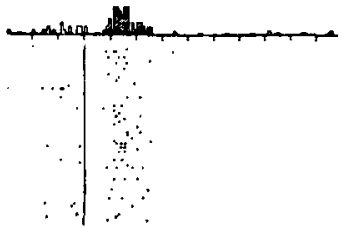

B

\section{OUTPUT}

STIMULUS CURRENT IN MOTOR CORTEX

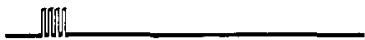

EDC TENSION

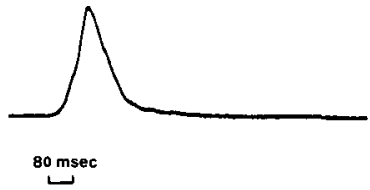

Figure 5-Input-output linkages in motor cortex. A. $80 \mu$ displacement of extensor digitorum communis (EDC) muscle produces short latency response in motor cortex neuron. PSTH time marker: $10 \mathrm{msec}$. Raster below PSTH shows individual spikes for each response, layered horizontally with

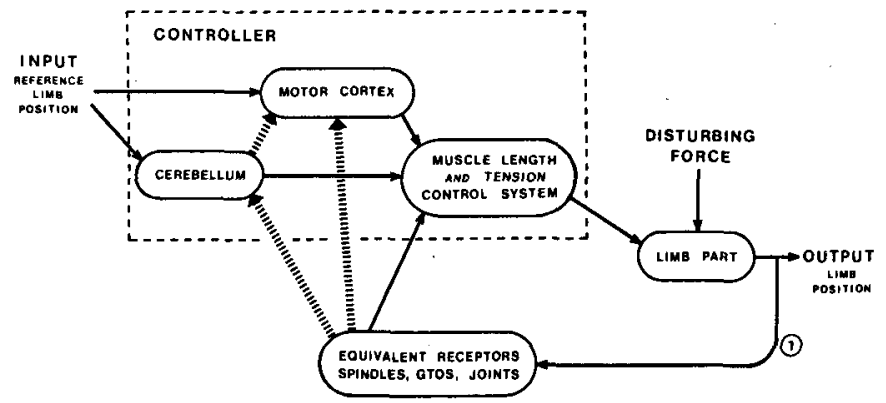

Figure 6-Feedback loops through cerebellum and motor cortex for limb position control system.

real time sequenced from above down. B. Stimulus current of $16 \mu \mathrm{A}$ amplitude is applied in train of pulses at $300 \mathrm{~Hz}$ (pulse frequency not drawn to scale); train duration of $80 \mathrm{msec}$. is indicated in time marker below tension trace. Amplitude of tension increment in EDC is $50 \mathrm{gm}$. wt. reticular formation; these pathways constitute additional feedback loops which probably introduce a further distribution of time delays.

To demonstrate that these feedback loops are actually connected to the output in the functionally intact animal, after reversing neuromuscular blockade we applied highly localized stimulating currents to the motor cortex. Stimulus currents were delivered through the same microelectrode from which recordings from single neurons responsive to feedback from muscle receptors were obtained; moreover for these stimulations the microelectrode remained in the location at which the responsive neuron was recorded. These cortical microstimulations excited a spatially restricted group of neurons and produced contraction of single muscles in the forelimb (Asanuma and Sakata, 1967; Murphy et al., 1975). In this experimental situation, the muscle which contracts is the same one from which the feedback arises (Murphy et al., 1975). An example is shown in figure 5. The control system in figure 3 can now be modified as in figure 6 to indicate the presence of these additional feedback loops.

\section{Is There Velocity Related Information in the Feedback?}

This question could also be addressed in straight-forward fashion. The technique used was to measure the spike train responses of a single neuron to application of a series of muscle stretches with different velocities but the same amplitudes. We observed that both Purkinje neurons in the cerebellar cortex and interpositus neurons (which are the output neurons of the cerebellum) responded only to change of position with time (velocity) and not to steady-state position (Murphy et al., 1973a; MacKay and Murphy, 1974). We also demonstrated by cooling the interpositus that these interpositus neurons projected to output cells of the motor cortex, probably through ventrolateral thalamus. In turn, the neurons of the motor cortex responded only to velocity in these open-loop experiments, and not position (or length) information on the feedback channel from single muscles (Murphy et al., 1975).

Examples of these results are shown in Figure 7. It is evident that velocity related information is encoded in the responses of these output cells in the interpositus nucleus and in the motor cortex (the latter identified by antidromic activation from stimulation in the medullary pyramid). It should be noted parenthetically that the independent, limniscal channel to the motor cortex also transmits velocity-related information. Our measurements indicate that the lemniscal channel has a higher sensitivity, but a smaller dynamic range than the transcerebellar channel; thus the two channels, transcerebellar and lemniscal, send complementary feedback information to the motor cortex.

One may enquire how velocity related information is encoded. Firstly, it is encoded originally at the receptor level, principally in primary endings of muscle spindles, but also in GTOs (Matthews, 1972). Secondly, feedforward and feedback inhibition is superimposed at every stage of the sequence through intermediate structures on the pathways. In most cases local inhibitory interneurons are responsible. However other mechanisms, for example, presynaptic inhibition and/or disfacilitation, are also known to exist. These inhibitory circuits can act as a "filter" by differentiating with respect to time any position or length information originating in the receptors. These filtering mechanisms must be extremely important in the case of secondary spindle endings which transmit length information in the first order afferent fibers, and which may project to the interpositus nucleus of the cerebellum (Kawaguchi and Ono, 1974; MacKay and Murphy, 1974).

\section{CONCLUSIONS}

These experiments provide indisputable answers to the simple questions posed. Firstly, multiple feedback loops to the CNS controller exist, and these act to distribute the 
feedback over time. Secondly, velocity-related information is carried in some of these feedback channels which offers the possibility of phase-leads in the feedback to compensate for time delays. Finally, the cerebellum constitutes a major part of one of these feedback channels.

We now return to the precepts of elementary control theory and predict that a lesion affecting those parts of the cerebellum participating in these feedback loops will result in a more unstable position control system than when the cerebellum is intact. This instability will be manifested in the form of oscillations when the system is perturbed. Perturbations can be generated by a peripheral stimulus ("disturbance" in figures 3 and 6), or internally by the engram subserving voluntary movement which is implicit in the "input" to the system. Verification of this prediction was provided more than half a century ago by the meticulous, quantitative observations of Gordon Holmes (1917); moreover Holmes' observations have recently been confirmed in a highly controlled experimental situation in primates (Brooks et al., 1973). The present elementary formulation thus answers a part of one of the major problems in neurobiology by showing that a principal function of the cerebellum is to act as a velocity feedback channel for the limb position control system of the central nervous system.

The recent closed-loop experiments on the vestibulo-ocular reflex in cats by D. A. Robinson (1974) are interesting in this context. He showed that the vestibulocerebellum also serves a stabilizing function by correcting the gain of this reflex. These observations conform to the general view that those parts of the cerebellum which receive afferent projections from the periphery (paleo- and archicerebellum) function as part of a feedback loop for a position control system. The neocerebellum, which has progressively developed in primates, appears to receive only "internal" feedback, from neocortex. The lateral (dentate) cerebellar nuc- leus, which is in large measure the output network for neocerebellum, modulates firing of neurons in the motor cortex during voluntary movements (Meyer-Lohman et al., 1975), and this modulation may also take the form of adjustments in gain of external feedback loops (Murphy et al., 1975).

The mossy fibers are the afferent system to the cerebellum which subserves the physiological feedback system described above. The function of the second afferent system, climbing fibers, is less precisely known. There is evidence that the climbing fiber system discharges at the termination of old movements and the beginning of new movements (Thach, 1971). In addition climbing fibers appear to have a resetting action on Purkinje cells in the cerebellar cortex (Murphy and Sabah, 1971). These two sets of observations suggest that the climbing fibers may be part of a "braking" subsystem for the limb position control system. This braking action could be initiated either from the motor cortex or from the periphery, since neurons in the inferior olive, which is a prime parent nucleus of climbing fibers, can be excited from these two sources (Crill, 1970; Sedgewick and Williams, 1967).

\section{A}

MOTOR CORTEX
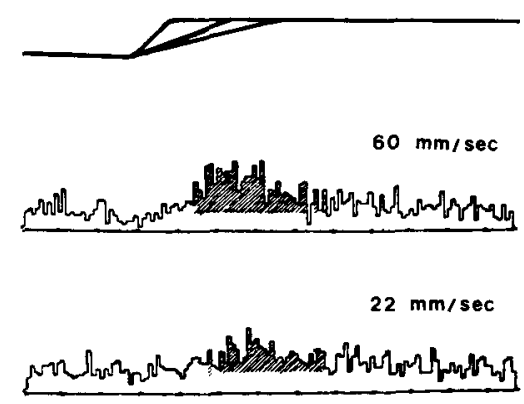

$17 \mathrm{~mm} / \mathrm{sec}$

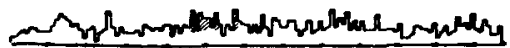

Additional evidence for this view of climbing fiber function has been provided by the studies of DeMontigny and Lamarre (1973) who showed that the climbing fiber system discharged with the same frequency as experimental tremor; the latter is enhanced by the drug Harmaline, which exerts its principal effect in the inferior olive.

It should be emphasized that our general knowledge of the internal operations of the limb position control system remains exceedingly scant. Further investigations may profitably be directed toward examination of other internal and external feedback loops, and of the degree of non-linearity present in various parts of the system.

\section{DISCUSSION}

Abrahams (Queens) drew attention to the probable importance of the roles of the three functional types of muscles and also to the locations of the spindles and Golgi tendon organs in relationship to slow muscle fibers, as well as to the tendinous insertions. Feedback during movement thus should contain information not only about length, velocity, or force exerted by the whole muscle, but also about more subtle sequential events in smaller muscle parts. Murphy in reply offered the observation that the signal-to-noise ratio about information from spindles becomes improved in records from neurones in the external cuneate nucleus, where convergence from

B

\section{INTERPOSITUS}

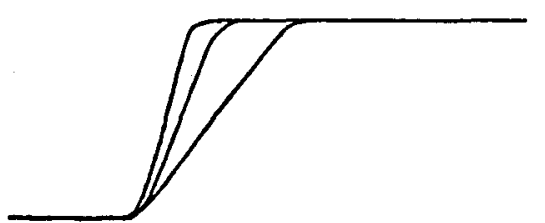

$153 \mathrm{~mm} / \mathrm{sec}$

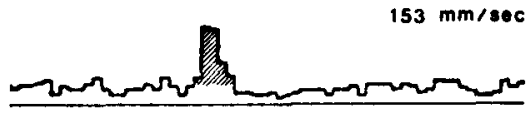

$109 \mathrm{~mm} / \mathrm{sec}$

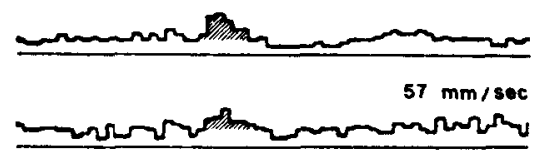

Figure 7-Velocity coding by single neurons in motor cortex (A) and cerebellar nucleus (B). Velocities of stretch of simple forelimb muslces are indicated above histogram. Total analysis time in $A$ and $B$ is $128 \mathrm{msec}$. Bin sizes: $1 \mathrm{msec}$. (A) and 2 msec. (B). 
about 16 primary afferent fibers has taken place.

Hore asked Murphy to confirm that the reduction of precentral responses to stretch in his experiments with cooling interpositus could be due to its disfacilitation, rather than to removal of afferent information through the interpositus route. Murphy agreed that information from Golgi tendon organs and muscle secondary spindles could become ineffective in that way but stressed that different populations of precentral neurons respond to signals from interpositus as opposed to medial lemniscus.

Hore concluded the exchange by pointing out that a limb perturbation causes a synchronous barrage of inputs from joints, skin and muscle, and that therefore high threshold muscle afferents may not be too important in generating the early cortical discharge reported in Brooks' presentation.

\section{ACKNOWLEDGEMENTS}

The authors' research is supported by the MRC of Canada. We thank Micheal O'Riain for reviewing the manuscript, and Jennifer Daynes and Hoi Nguyen-Huy for expert technical and programming assistance.

\section{REFERENCES}

ASANUMA, H. and SAKATA, H. (1967). Functional organization of a cortical efferent system examined with focal depth stimulation in cats. J. Neurophysiol., 30, 35-54.

BRODAL, A. (1967). Anatomical studies of cerebellar fibre connections with special reference to problems of functional localization. In: The Cerebellum. Progress in Brain Research, Vol. 25, C. A. Fox and R. S. Snider (eds.), pp. 135-173, Elsevier, Amsterdam.

BROOKS, V. B., KOZLOVSKAYA, I. B., ATKIN, A., HORVATH, F. E. and UNO, M. (1973). Effects of cooling dendate nucleus on tracking-task performance in monkeys. J. Neurophysiol., 36, 974-995.

CRILL, W. E. (1970). Unitary mutiplespiked responses in cat inferior olive nucleus. J. Neurophysiol., 33, 199-209.

DEMONTIGNEY, C. and LAMARRE, Y. (1973). Rhythmic activity induced by harmaline in the olivo-cerebello-bulbar system of the cat. Brain Research, 53, 81-95.
ECCLES, J. C. (1964). The Physiology of Synapses, pp. 316, Springer-Verlag, Heidelberg.

ECCLES, J. C., FABER, D. S., MURPHY, J. T., SABAH, H. and TABORIKOVA, $H$. (1971). Investigations on integration of mossy fiber inputs to Purkyne cells in the anterior lobe. Exp. Brain Res., 13, 54-77.

GRANT, G. (1962). Spinal course and somatotopically localized termination of the spinocerebellar tracts. An experimental study in the cat. Acta Physiol. Scand. 56, supp. 193, 5-61.

HOLMES, G. (1917). The symptoms of acute cerebellar injuries due to gunshot injuries. Brain, 40 (4), 461-535.

HOLMES, G. (1922). The Croonian lectures on the clinical symptoms of cerebellar disease and their interpretation. The Lancet, 202, 1231-1237.

HOLMES, G. (1939). The cerebellum of man. Brain, 62, 1-30.

HOUK, J. and HENNEMAN, E. (1974). Feedback control of muscle: introductory concepts. in: Medical Physiology, Vol. 13, V. B. Mountcastle (ed.), pp. 608-616, Mosby, St. Louis.

KAWAGUCHI, S. and ONO, T. (1974). Responses on interpositus neurones to inputs from muscle receptors. Exptl. Brain Res., $21,375-386$.

LUCIER, G. E., RUEGG, D. G., and WIESENDANGER, M. (1975, in press). Responses of neurones in motor cortex and in area $3 \mathrm{a}$ to controlled stretches of forelimb muscles in cebus monkeys. J. Physiol.

MacKAY, W. A. and MURPHY, J. T. (1974). Responses of interpositus neurons to passive muscle stretch. J. Neurophysiol., 37, 1410-1423.

MATTHEWS, P. B. C. (1972). Mammalian Muscle Receptors and their Central Actions, 630 pp., Arnold, London.

MEYER-LOHMANN, J., CONRAD, B., MATSUNAMI, $K$, and BROOKS, V. B. (1975, in press). Effects of dentate cooling on precentral unit activity following torque pulse injections into elbow movements. Brain Research.

MURPHY, J. T., MacKAY, W. A. and JOHNSON, F. (1973a). Responses of cerebellar cortical neurons to dynamic proprioceptive inputs from forelimb muscles. J. Neurophysiol., 34, 711-723.

MURPHY, J. T., MacKAY, W. A. and JOHNSON, F. (1973b). Differences between cerebellar mossy and climbing fibres responses to natural stimulation of forelimb muscle proprioceptors. Brain Research, 55, 263-289.

MURPHY, J. T. and SABAH, N. H. (1971). Cerebellar Purkinje cell responses to afferent inputs: I. Climbing fiber activation. Brain Research, 25, 449-467.

MURPHY, J. T., WONG, Y. C. and KWAN, H. C. (1975, in press). Afferentefferent linkages in motor cortex for single forelimb muscles. J. Neurophysiol.

OSCARSSON， O. (1967). Functional significance of information channels from the spinal cord to the cerebellum. In: Neurophysiological Basis of Normal and Abnormal Motor Activities. M. D. Yahr and D. P. Purpura (eds.), pp. 93-116 Raven Press, New York.

ROBINSON, D. A. (1974). Cerebellar adaptation of the vestibuloocular reflex to modified visual input. Soc. for Neuroscience, 4th Annual Meeting, St. Louis, p. 397.

ROSEN, I. (1972). Projection of forelimb group I muscle afferents to the cat cerebral cortex. Intl. Rev. Neurobiol.; 15, 1-25.

SEDGEWICK, E. M. and WILLIAMS, T. D. (1967). Responses of single units in the inferior olive to stimulation of the limb nerves, peripheral skin receptors, cerebellum, caudate nucleus, and motor cortex. J. Physiol., 189, 261-279.

STEIN, R. B. (1974). Peripheral control of movement. Physiol. Rev., 54, 215-243.

THACH, W. T. (1970). Discharge of cerebellar neurons to two maintained postures and two prompt movements. II. Purkinje cell output and input. J. Neurophysiol., 33, 537-546.

VOOGD, J., BROERE, G. and VAN ROSSUM, J. (1969). The medio-lateral distribution of the spinocerebellar projection in the anterior lobe and the simple lobule in the cat and a comparison with some other afferent fibre systems. Psychiat. Neurol. Neurochir., 72, 137-151.

WIENER, N. (1948). Cybernetics, pp. 194, Wiley, New York. 